Original Article

\title{
Effects of two-handed task training on upper limb function of chronic hemiplegic patients after stroke
}

\author{
Byung IL YANG ${ }^{1)}$, Bo Kyoung Song ${ }^{2)}$, SANG Mi Joung ${ }^{3)^{*}}$ \\ 1) Department of Physical Therapy, Bobath Memorial Hospital, Republic of Korea \\ 2) Department of Occupational Therapy, Kangwon University, Republic of Korea \\ 3) Department of Occupational Therapy, Sanggi Youngseo University: 84 Sanggidae-gil, Wonju-si, \\ Gangwon-do 26339, Republic of Korea
}

\begin{abstract}
Purpose] The purpose of this study was to determine whether two-handed task training is effective on motor learning of injured cerebral cortex activation and upper extremity function recovery after stroke. [Subjects and Methods] Two hemiplegic subjects participated in this study: one patient was affected on the dominant side of the body and the other was affected on the non-dominant side of the body, and both scored in the range of 58-66 in the Fugl-Meyer assessment. The excitability of the corticospinal tract and Manual Function Test were examined. [Results] The excitability of the corticospinal tract and the Manual Function Test showed significant differences in the activation of both sides of the cerebral cortex and in the variation in learning effect of upper extremity motor function recovery in patients with hemiplegic non-dominant hand (left). [Conclusion] The results suggested that two-handed task training had a different influence on dominant hand (right) and non-dominant hand (left) motor recovery.

Key words: Chronic stroke, Dominant hand, Two-handed task training
\end{abstract}

(This article was submitted Aug. 16, 2016, and was accepted Oct. 11, 2016)

\section{INTRODUCTION}

The dominant hand is defined as the hand that is usually used in performing activities of daily living (ADL). The development of the motor function of the cerebral cortex is asymmetrical to the dominant hand ${ }^{1)}$. Based on such asymmetrical development of the cerebral cortex, when the left hand is performing a task, the cerebral cortex motor area of the right cerebral hemisphere activates. However, a more interesting fact is that when the right hand is used functionally, the nerve cells of the entire cerebral cortex motor area of the right and left cerebral hemispheres activate ${ }^{2)}$. This finding supports the evidence of lateralization of the cerebral hemisphere and implies that the left cerebral hemisphere acts the role of the dominant cerebral hemisphere when performing $\mathrm{ADL}^{2,3)}$. Thus, the left hemisphere, which is the dominant cerebral hemisphere due to the lateralization of the cerebral hemisphere, is more closely related with motor planning in ADL performance, and the same relationships were shown after cerebral hemisphere injury due to stroke ${ }^{3,4)}$. Characteristically, the patient with stroke-damaged dominant left cerebral hemisphere reports a time delay on the exercise performance of both right and left hands, whereas the patient with damaged right cerebral hemisphere reports a mild motor function disorder confined to the left hand ${ }^{3}$. This means that, consequently, after stroke onset, the patient with hemiplegic dominant hand (right) experiences more difficulty in performing $\mathrm{ADL}^{5}$. . However, regarding upper extremity rehabilitation, there is no study that differentiated the motor function recovery of the upper extremity of patients who are affected in the dominant cerebral hemisphere (with the hemiplegic right hand) or in the non-dominant cerebral hemisphere (with the hemiplegic left hand). A specific rehabilitation

\footnotetext{
*Corresponding author. Sang Mi Joung (E-mail: otjsm@hanmail.net)

(C)2017 The Society of Physical Therapy Science. Published by IPEC Inc.

This is an open-access article distributed under the terms of the Creative Commons Attribution Non-Commercial No Derivatives (by-nc-nd) License $<$ http://creativecommons.org/licenses/by-nc-nd/4.0/>.
} 
approach based on laterality through classification of cerebral damage on the right or left side is needed to achieve a more successful rehabilitation of the upper extremity. Thus, the purpose of this study is to determine whether two-handed task training is effective in the motor learning of the injured cerebral cortex activation and upper extremity function recovery after stroke.

\section{SUBJECTS AND METHODS}

Two subjects with hemiplegia participated in this study. One patient was affected on the dominant side of the body and the other patient was affected on the non-dominant side of the body. Patients with diagnosed stroke (hemorrhagic/infarction) $>6$ months, with no cognitive impairment (Mini Mental State Examination, MMSE-K $\geq 24$ ), with $>58$ points on the FuglMeyer motor assessment of upper extremities ${ }^{6}$ were included in the study. The exclusion criteria of this study were severely impaired internal carotid artery, intracranial metallic implant, and/or seizure. The subjects were outpatients in Seongnam B Rehabilitation Hospital. This study followed the ethical principles of the Declaration of Helsinki, and all patients gave written informed consent before participating in this study. A MagPro R30 transcranial magnetic stimulation (TMS) equipment (Medtronic, Skovlunde, Denmark) was used to measure the changes in excitability of the corticospinal tract. To determine the exact location of the motor cortex area of the first dorsal interosseous (FDI), we used a single stimulus, moving the location on the head of subject using a coil stimulator. In the FDI electrograph, the point where the biggest motor evoked potential (MEP) appeared was considered as the motor cortex area. The resting motor threshold was defined as the least stimulus strength from which $>50 \mu \mathrm{V}$ was recorded at least more than 5 out of 10 stimuli; the amplitude and latency values of the MEP that was stimulated by $120 \%$ were measured 15 times and averaged ${ }^{7}$. In addition, the Manual Function Test was used to determine the overall function of the upper extremity ${ }^{8}$. The two subjects were trained twice a week, for 6 weeks (total, 12 times). After the training ended, the two subjects underwent upper extremity function reassessment; each subject was reassessed once and analyzed 12 times in total for possession examinations 2 weeks later and 3 months later. Every subject chose the functional task training that uses a two-handed task often in everyday life as the therapeutic intervention of upper extremity rehabilitation. The two-handed tasks training program included the following exercises: (1) washing of hands: hand washing using real soap in a basin in the treatment room, with the therapist offering a bit of support as needed; (2) putting on clothes: putting on the top half of a patient gown after getting undressed, with the therapist offering a bit of support as needed; (3) scissoring: using a certain size of scissors, the patient cuts a thick sheet of A4-size paper, with the therapist offering a bit of support as needed; (4) opening a bottle cap: this involves opening a soda bottle cap using a bottle opener, with the therapist offering a bit of support as needed; (5) muscular strength training: training both hands using two $\mathrm{JAMAR}^{\circledR}$ hand dynamometer, with the therapist offering a bit of support as needed. Each task was performed for 6 minutes, and all tasks were accomplished in 30 minutes; the order of tasks was random. A graph was used to analyze the effect of the two-handed task with or without a dominant hand deficit on cerebral cortex activation and upper extremity function recovery.

\section{RESULTS}

The MEP amplitude in subject 1 (with the hemiplegic dominant hand [right]) increased from $0.13 \mathrm{mV}$ in the pre-training period to $0.27 \mathrm{mV}$ after 12 sets of training, to $0.25 \mathrm{mV}$ after 2 weeks, and to $0.24 \mathrm{mV}$ after 3 months (Table 1). The latency period decreased from $24.23 \mathrm{~ms}$ in the pre-training period to $23.03 \mathrm{~ms}$ after a total of 12 sets of training, to $23.5 \mathrm{~ms}$ after 2 weeks, and to $23.72 \mathrm{~ms}$ after 3 months (Table 2). The average of the MEP amplitude in subject 2 (with the hemiplegic nondominant hand [left]) increased from $0.36 \mathrm{mV}$ in the pre-training period to $0.46 \mathrm{mV}$ after 12 sets of training, to $0.44 \mathrm{mV}$ after 2 weeks, and to $0.42 \mathrm{mV}$ after 3 months (Table 1). The latency period decreased from $24.93 \mathrm{~ms}$ in the pre-training period to $24.44 \mathrm{~ms}$ after a total of 12 sets of training, to $24.35 \mathrm{~ms}$ at the possession examination after 2 weeks, and to $24.6 \mathrm{~ms}$ at the possession examination after 3 months (Table 2). MFT was used to assess both subjects 1 and 2 at base terms, i.e., the terms of arbitration and possession examination of training of both hands. The average MFT of subject 1 increased from 19.3 to 21 points and was maintained at 21 points at the possession examination conducted after 2 weeks and 3 months (Table 3 ). The average MFT of subject 2 increased from 25 to 26 points after the training ended and was maintained at 26 points at the possession examination conducted after 2 weeks and 3 months (Table 3 ).

\section{DISCUSSION}

This study was conducted to investigate, in the context of two-handed task training, how therapeutic intervention in patients with hemiplegic dominant hand (right) and hemiplegic non-dominant hand (left) affects both sides of cerebral cortex activation and the variation of learning and upper extremity motor function recovery, since most tasks in daily life require the use of both hands. According to a previous study using TMS, representation can be effected on the primary motor area (M1) of the cerebral cortex, and the MEP values obtained through TMS can confirm the changes in relevant areas more accurately and effectively ${ }^{9}$. Characteristically, some features such as the hemiplegic side showing higher stimulus threshold, lower MEP values, and delayed MEP latency period, compared with the non-hemiplegic side, were observed ${ }^{9-11)}$. This study repeatedly measured the variables expressed in the M1 area of the cerebral cortex to determine the average MEPs 
Table 1. Changes in the motor-evoked potential amplitude (mV) of subjects 1 and 2

\begin{tabular}{|c|c|c|c|c|c|c|c|c|c|c|c|}
\hline & \multicolumn{3}{|c|}{ Base } & \multicolumn{6}{|c|}{ Intervention } & \multicolumn{2}{|c|}{ Retention } \\
\hline & 1 & 2 & 3 & 4 & 5 & 6 & 7 & 8 & 9 & 10 & 11 \\
\hline Subject 1 (dominant) & 0.13 & 0.12 & 0.13 & 0.12 & 0.16 & 0.19 & 0.18 & 0.22 & 0.27 & 0.25 & 0.24 \\
\hline Subject 2 (non-dominant) & 0.36 & 0.35 & 0.36 & 0.43 & 0.46 & 0.46 & 0.45 & 0.47 & 0.46 & 0.44 & 0.42 \\
\hline
\end{tabular}

Table 2. Changes in the motor-evoked potential latency (msec) of subjects 1 and 2

\begin{tabular}{|c|c|c|c|c|c|c|c|c|c|c|c|}
\hline & \multicolumn{3}{|c|}{ Base } & \multicolumn{6}{|c|}{ Intervention } & \multicolumn{2}{|c|}{ Retention } \\
\hline & 1 & 2 & 3 & 4 & 5 & 6 & 7 & 8 & 9 & 10 & 11 \\
\hline Subject 1 (dominant) & 24.2 & 24.3 & 24.2 & 23.59 & 23.477 & 23.49 & 23.24 & 23.32 & 23.03 & 23.5 & 23.72 \\
\hline Subject 2 (non-dominant) & 25 & 24.97 & 24.83 & 25.01 & 24.57 & 24.61 & 24.68 & 24.49 & 24.44 & 24.35 & 24.6 \\
\hline
\end{tabular}

Table 3. Changes in the Manual Function Test (MFT) score of subjects 1 and 2

\begin{tabular}{|c|c|c|c|c|c|c|c|c|c|c|c|}
\hline & \multicolumn{3}{|c|}{ Base } & \multicolumn{6}{|c|}{ Intervention } & \multicolumn{2}{|c|}{ Retention } \\
\hline & 1 & 2 & 3 & 4 & 5 & 6 & 7 & 8 & 9 & 10 & 11 \\
\hline Subject 1 (dominant) & 19 & 19 & 20 & 19 & 19 & 21 & 21 & 21 & 21 & 21 & 21 \\
\hline Subject 2 (non-dominant) & 25 & 25 & 25 & 25 & 26 & 26 & 26 & 26 & 26 & 26 & 26 \\
\hline
\end{tabular}

and delayed latency time of the affected side using TMS to check for upper extremity motor function learning in stroke patients. The increased average MEPs of the cerebral cortex and the reduction in delayed latency time in both subjects 1 and 2 were confirmed through the comparison of values before and after training. These results provide support for research that intensive and repetitive upper extremity functional training can improve brain plasticity of cerebral cortex brain regions in stroke patients ${ }^{11)}$. Furthermore, this study ascertained that intensive and repetitive upper extremity function training with and without the use of the hemiplegic dominant hand shows a difference in cerebral cortex activation. In contrast, when the task training involved both hands, no difference in cerebral cortex activation takes place. In this study, comparison of the two subjects before and after training confirmed the increase in average MEPs and the decrease in delayed latency time in the patient with hemiplegic dominant hand. This suggest that the same task training using both hands may result in a difference in the restoration of upper extremity function depending on the use of the hemiplegic dominant hand in stroke patients. This study also shows an increased activity in the left cerebral cortex (dominant hand) rather than the right cerebral cortex (nondominant hand) in the task training of both hands in functional ADL, which can lead to better motor recovery in patients with right hemiplegia (dominant hand). The results show the possible function of the corpus callosum, which connects the M1 or supplementary motor area with the interhemispheric area, in increasing the activity of the damaged cerebral hemisphere when task training with both hands ${ }^{12}$. In addition, this study has determined that the activation of the left cerebral cortex (dominant hand) was further increased by task training using both hands. The frequency of using the right hand at a given time is much higher than that of the left hand, even if the task was performed with both hands, because the right hand plays a role in mobility, whereas the left is for stability. These have the same context as the report that showed that the activation of the cerebral cortex on functional magnetic resonance imaging increases with increasing rate of movement ${ }^{12,13)}$. Although this study showed a change in trend while the two subjects (one dominant hand [right] and one non-dominant hand [left]) were performing, hemisphere dominance was difficult to generalize statistically because of the small number of subjects. This study on two-handed task performance was conducted with ADL in mind, not only stereotyped paradigm inducing bilateral symmetrical movements, and identified changes in cerebral cortex activation and upper extremity functional recovery in stroke patients according to dominant hand. In the future, a specific upper extremity rehabilitation approach is necessary, one that recognizes movement difference between the dominant and non-dominant hands by considering the different movement dominance of the right and left hemispheres.

\section{REFERENCES}

1) De Gennaro L, Cristiani R, Bertini M, et al.: Handedness is mainly associated with an asymmetry of corticospinal excitability and not of transcallosal inhibition. Clin Neurophysiol, 2004, 115: 1305-1312. [Medline] [CrossRef]

2) Kim SG, Ashe J, Hendrich K, et al.: Functional magnetic resonance imaging of motor cortex: hemispheric asymmetry and handedness. Science, 1993, 261: 
615-617. [Medline] [CrossRef]

3) Sabaté M, González B, Rodríguez M: Brain lateralization of motor imagery: motor planning asymmetry as a cause of movement lateralization. Neuropsychologia, 2004, 42: 1041-1049. [Medline] [CrossRef]

4) Zemke AC, Heagerty PJ, Lee C, et al.: Motor cortex organization after stroke is related to side of stroke and level of recovery. Stroke, 2003, 34: e23-e28. [Medline] [CrossRef]

5) Sainburg RL, Duff SV: Does motor lateralization have implications for stroke rehabilitation? J Rehabil Res Dev, 2006, 43: 311-322. [Medline] [CrossRef]

6) Sullivan KJ, Tilson JK, Cen SY, et al.: Fugl-Meyer assessment of sensorimotor function after stroke: standardized training procedure for clinical practice and clinical trials. Stroke, 2011, 42: 427-432. [Medline] [CrossRef]

7) Rossini PM, Barker AT, Berardelli A, et al.: Non-invasive electrical and magnetic stimulation of the brain, spinal cord and roots: basic principles and procedures for routine clinical application. Report of an IFCN committee. Electroencephalogr Clin Neurophysiol, 1994, 91: 79-92. [Medline] [CrossRef]

8) Miyamoto S, Kondo T, Suzukamo Y, et al.: Reliability and validity of the Manual Function Test in patients with stroke. Am J Phys Med Rehabil, 2009, 88 : 247-255. [Medline] [CrossRef]

9) Stinear CM, Barber PA, Smale PR, et al.: Functional potential in chronic stroke patients depends on corticospinal tract integrity. Brain, 2007, 130: 170-180. [Medline] [CrossRef]

10) Rossini PM, Pauri F: Neuromagnetic integrated methods tracking human brain mechanisms of sensorimotor areas 'plastic' reorganisation. Brain Res Brain Res Rev, 2000, 33: 131-154. [Medline] [CrossRef]

11) Schaechter JD: Motor rehabilitation and brain plasticity after hemiparetic stroke. Prog Neurobiol, 2004, 73: 61-72. [Medline] [CrossRef]

12) Staines WR, McIlroy WE, Graham SJ, et al.: Bilateral movement enhances ipsilesional cortical activity in acute stroke: a pilot functional MRI study. Neurology, 2001, 56: 401-404. [Medline] [CrossRef]

13) Rao SM, Bandettini PA, Binder JR, et al.: Relationship between finger movement rate and functional magnetic resonance signal change in human primary motor cortex. J Cereb Blood Flow Metab, 1996, 16: 1250-1254. [Medline] [CrossRef] 